\title{
Utilisation de la relaxation chimique pour l'étude des interactions entre protéines
}

\author{
Jean-Michel El Hage, Miryana Hemadi, Nguyêt-Thanh Ha-Duong
}

ITODYS, Université Paris 7, Paris

\begin{abstract}
La relaxation chimique correspond au réajustement d'un système de réactions chimiques réversibles écartées de l'équilibre par une faible perturbation, saut de température ou de concentration par exemple. Les techniques expérimentales actuelles permettent de mesurer par spectroscopie des cinétiques de réaction correspondant à des temps de relaxation pouvant varier de la microseconde à quelques jours. Nous les illustrons par un exemple d'analyse des interactions entre deux protéines, la transferrine et son récepteur membranaire, responsables du transport et de l'acquisition du fer chez les vertébrés, et montrons que l'on peut ainsi identifier les différentes étapes de la réaction.
\end{abstract}

Les méthodes et techniques de la relaxation chimique ont été mises au point et développées à partir des années cinquante par M. Eigen (prix Nobel de chimie, 1967) dans le but d'analyser la vitesse des réactions rapides ou ultra-rapides [1]. Eigen a été le premier à mesurer le temps de dissociation de l'eau $\left(<10^{-6}\right.$ seconde) et à rapporter des constantes cinétiques de transferts de proton contrôlés par la diffusion $\left(\sim 10^{11} \mathrm{M}^{-1} \mathrm{~s}^{-1}\right)$. Depuis, a été accompli un travail colossal sur pratiquement tous les aspects des réactions chimiques réversibles dites rapides ou ultrarapides. Des centaines de réactions de proto-dissociation, de tautomérie, d'interactions moléculaires, de micellisation, de formation de complexes métalliques avec des dizaines de métaux et des centaines de ligands, et de nombreux autres types de réactions ont été et continuent à être analysées par les techniques et méthodes de la relaxation chimique. Eigen a été, par ailleurs, celui qui a déclenché la course à la détection et l'analyse des réactions ultra-rapides. Cette course a abouti à l'émergence de la " femtochimie » [2], qui permet depuis les années quatre-vingt de mesurer la vitesse des processus élémentaires se déroulant à l'échelle de la femtoseconde ( $10^{-15}$ seconde).

Nous allons introduire sommairement les principes de la relaxation chimique et les appliquer aux mécanismes d'interaction entre les protéines responsables du transport et de l'acquisition du fer chez les vertébrés: les transferrines et leurs récepteurs [3, 4].

\section{Cinétique de relaxation, linéarisation des équations de vitesse}

Tout équilibre chimique est affaire de dynamique : $A$ et $B$ réagissent constamment pour donner $C$, et $C$ se dissocie constamment en $\mathrm{A}$ et $\mathrm{B}$ (équation 1).

$$
\mathrm{A}+\mathrm{B} \underset{\mathrm{k}_{-1}}{\stackrel{\mathrm{k}_{1}}{\rightleftharpoons}} \mathrm{C}
$$

où $\mathrm{k}_{1}$ et $\mathrm{k}_{-1}$ sont les constantes cinétiques élémentaires, respectivement de premier et second ordre. L'équation cinétique de la réaction (1) s'écrit :

$$
-d[A] / d t=-d[B] / d t=d[C] / d t=k_{1}[A][B]-k_{-1}[C]
$$

À l'équilibre thermodynamique, la vitesse de la réaction est nulle $(d[A] / d t=0)$ et on peut écrire :

$$
\begin{aligned}
& \mathrm{k}_{1}[\mathrm{~A}][\mathrm{B}]=\mathrm{k}_{-1}[\mathrm{C}] \\
& {[\mathrm{A}][\mathrm{B}] /[\mathrm{C}]=\mathrm{k}_{-1} / \mathrm{k}_{1}=\mathrm{K}_{1}}
\end{aligned}
$$

où $\mathrm{K}_{1}$ est la constante d'équilibre.

Dans son aspect le plus général, la relaxation traite du réajustement d'un système de réactions chimiques écartées de l'équilibre par une faible perturbation. Un équilibre chimique peut dépendre d'un certain nombre de variables extrinsèques, telles que la température et la pression, ou intrinsèques telles que les concentrations. Si nous perturbons une de ces variables, nous perturberons aussi le système. Illustrons cette approche par le principe du saut de température, qui consiste à augmenter la température du système réactionnel de sa valeur initiale $T_{\mathrm{i}}$, où la constante d'équilibre s'exprime en fonction des concentrations à l'équilibre initial $\left(K_{i}=[A]_{i}[B]_{i} /[C]_{i}\right)$, à une valeur finale $T_{f}$, où la nouvelle constante d'équilibre s'exprime en fonction des concentrations à l'équilibre final $\left(K_{f}=[A]_{f}[B]_{f} /[C]_{f}\right)$. Cette augmentation de température doit se faire telle que le temps de montée de la perturbation soit le plus rapide possible (instantané) devant le temps de demi-vie de la réaction. II faut aussi que $T_{f}$ soit maintenue constante durant la réaction (figure 1). Que va-t-il se produire?

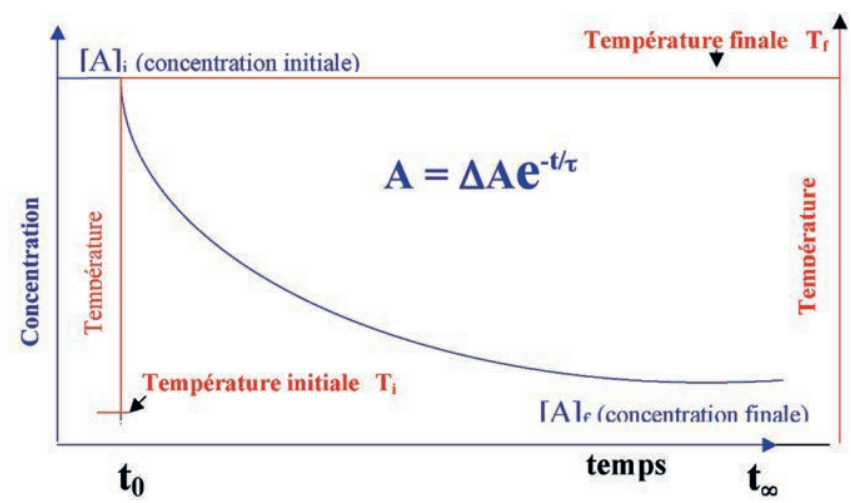

Figure 1. Illustration du rétablissement de l'équilibre d'un système chimique réversible après un saut instantané de température de $T_{j}$ à $T_{f}$. Réarrangement exponentiel des concentrations en fonction du temps.

Au temps $t=0$ : la température transite instantanément de $T_{i}$ à $T_{f}, K_{i}$ devient instantanément $K_{f}$, et se maintient tant que $T_{f}$ est stable. Toutefois, à $t=0$, la réaction n'a pas encore eu lieu et les concentrations des espèces présentes sont encore celles obéissant à $K_{i}\left([A]_{i},[B]_{i}\right.$ et $\left.[C]_{j}\right)$. Pour respecter la nouvelle constante d'équilibre $K_{f}$, ces concentrations vont se réarranger au cours du temps en $[A]_{f},[B]_{f}$ et $[C]_{f}$. Les concentrations au temps $t,[A]$, $[B]$ et $[\mathrm{C}]$ peuvent alors s'écrire : $[\mathrm{A}]=[\mathrm{A}]_{\mathrm{f}}+\Delta[\mathrm{A}],[\mathrm{B}]=[\mathrm{B}]_{\mathrm{f}}+\Delta[\mathrm{B}],[\mathrm{C}]$ $=[\mathrm{C}]_{\mathrm{f}}+\Delta[\mathrm{C}]$, où $\Delta[\mathrm{A}], \Delta[\mathrm{B}]$ et $\Delta[\mathrm{C}]$ sont les écarts de ces concentrations par rapport aux concentrations finales. La conservation de la masse implique que : $\Delta[\mathrm{A}]=\Delta[\mathrm{B}]=-\Delta[\mathrm{C}]$.

Si les variations des concentrations entre l'état initial et l'état final sont faibles, l'équation de vitesse (2) peut être linéarisée et devient :

$$
\begin{aligned}
& -\mathrm{d} \Delta[\mathrm{A}] / \mathrm{dt}=\left\{\mathrm{k}_{1}\left([\mathrm{~A}]_{\mathrm{f}}+[\mathrm{B}]_{\mathrm{f}}\right)+\mathrm{k}_{-1}\right\} \Delta[\mathrm{A}]=\tau^{-1} \Delta[\mathrm{A}] \\
& \text { La solution de cette équation s'écrit : } \\
& \quad \Delta[\mathrm{A}]=\Delta[\mathrm{A}]_{0} \exp (-\mathrm{t} / \tau)
\end{aligned}
$$

où $\Delta[\mathrm{A}]_{0}=[\mathrm{A}]_{\mathrm{i}}-[\mathrm{A}]_{\mathrm{f}}$ est l'amplitude de relaxation et $\tau$ le temps de relaxation.

La faible perturbation éloigne peu la réaction de sa position d'équilibre, ce qui, indépendamment de l'ordre de la réaction, réduit l'équation cinétique à une relation linéaire. Dans le cas des systèmes comprenant plusieurs réactions chimiques, l'évolution en fonction du temps des concentrations impliquées dans cha- 
cune de ces réactions devient alors une exponentielle pure (figure 1). Ainsi, les techniques de relaxation, quand elles sont applicables, simplifient considérablement l'analyse cinétique des systèmes chimiques complexes.

\section{Techniques de la relaxation chimique}

Le type de perturbation le plus utilisé est le saut de température par effet joule. Dans les appareils commerciaux, le saut de température est produit par la décharge rapide dans le milieu réactionnel d'un condensateur dans une cellule spécifique munie de deux électrodes en métal précieux (or ou platine) et de fenêtres optiques. Dans ces conditions et en fonction de la conductivité du milieu réactionnel, un saut de température de $15 \mathrm{~K}$ peut s'effectuer en 200 ns et la température finale reste constante pendant quelques $\mathrm{ms}$. La détection du réarrangement des concentrations se fait par spectrophotométrie d'absorption ou d'émission. Les signaux expérimentaux sont acquis et accumulés sur ordinateur.

La relaxation chimique n'est pas seulement réservée aux réactions rapides. II a été montré que dans certaines conditions expérimentales, elle est parfaitement applicable à l'analyse des cinétiques moyennement rapides ou lentes. Dans ces deux cas, la perturbation est induite par le saut d'une ou de plusieurs concentrations des espèces jouant un rôle actif dans le système. Ce saut de concentration peut être effectué en $2 \mathrm{~ms}$ avec un appareil d'« Écoulement Interrompu » (Stopped-Flow). Dans cet appareil, deux solutions sont mélangées, sous pression, d'une manière tangentielle aux parois d'une chambre de mélange spéciale munie de fenêtres de détection. L'écoulement est interrompu juste après le mélange avec un système de blocage mécanique qui déclenche aussi l'acquisition. La détection est le plus souvent spectrophotométrique. Pour les réactions lentes (> 10 secondes), le mélange est effectué en quelques secondes dans une cuve de spectrophotomètre par simple agitation. II faut souligner ici que pour pouvoir mesurer convenablement un temps de relaxation, il faut que le temps de montée de la perturbation soit au moins cinq fois plus rapide que le temps de relaxation lui-même (figure 1).

Nous disposons ainsi, avec les techniques de saut de température et de mélanges rapide ou lent, d'un éventail de méthodes nous permettant d'analyser des systèmes chimiques composés d'un ensemble de réactions dont les temps de relaxation peuvent varier d'une microseconde à quelques heures, voire quelques jours.

\section{Application de la relaxation chimique aux interactions protéine-protéine rapides et lentes, cas de la transferrine et de son récepteur $[3,4]$}

La sérum-transferrine humaine est le transporteur naturel du fer dans le sang. Quand la protéine est chargée en fer sous forme de holotransferrine, elle est reconnue par son récepteur membranaire et forme un adduit (produit issu d'une réaction d'addition) protéine-protéine. Le couple holotransferrine-récepteur est alors véhiculé à l'intérieur de la cellule dans un sac membranaire appelé endosome. Dans le cytoplasme, les endosomes contenant le couple transferrine chargée en fer/récepteur s'acidifient. Le fer est alors libéré et le nouvel adduit récepteur-apotransferrine (transferrine libre de fer), recyclé vers la surface de la cellule (figure 2).

La transferrine est une protéine bilobale, dont chaque lobe possède une cavité de fixation du fer (III) dans laquelle le métal est enfoui à $\sim 10 \AA ̊$ de la surface de la molécule (figure $3 A)$. Le récepteur membranaire de la transferrine est une protéine homodimérique formée de deux Figure 3: (A) Structure type "ruban " d'une sérum-transferrine humaine unité possède une partie de la sérum-transferrine humaine.

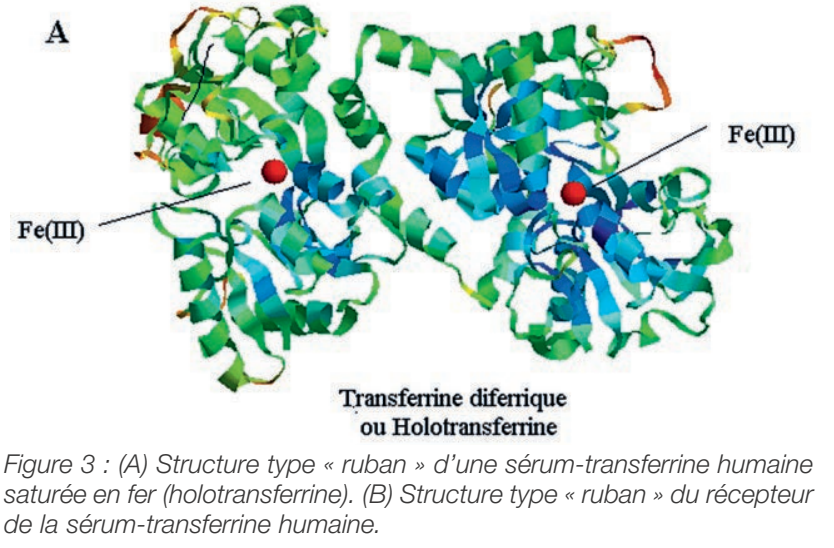

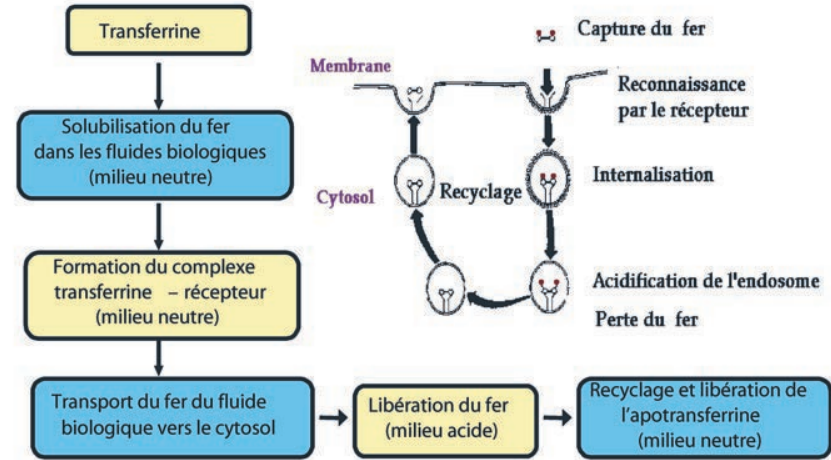

Figure 2 : Voie principale d'acquisition du fer chez les mammifères. Endocytose (incorporation dans la cellule) du couple holotransferrine-récepteur de la transferrine et recyclage de l'apotransferrine vers la surface cellulaire.

transmembranaire et un ectodomaine dirigé vers le milieu aqueux. Le récepteur a la forme d'un papillon dont les ailes constituent une forme apicale, le corps une forme hélicoïdale et dont la partie proche de la membrane forme avec cette dernière une cavité d'une cinquantaine d'angströms (figure 3B). Les différences de structures entre la transferrine et son récepteur font que ces deux protéines possèdent des spectres caractéristiques d'absorption et d'émission qui vont permettre la détection de leur réarrangement au cours du temps pendant les mesures cinétiques. Pour des raisons techniques, le récepteur de la transferrine a été solubilisé dans des micelles d'un détergent, le CHAPS, qui jouent ici le rôle d'une membrane artificielle.

L'application des méthodes et techniques de la relaxation chimique au système transferrine-récepteur-CHAPS a permis d'établir le mécanisme d'interaction de la transferrine avec son récepteur.

Quand une solution de récepteur de transferrine dans un milieu neutre et en présence de CHAPS est soumise à un saut de température rapide $(200 \mathrm{~ns})$ de 25 à $37{ }^{\circ} \mathrm{C}$, un seul processus cinétique est détecté (figure $4 \mathrm{~A}$ ). Ce phénomène, ultra-rapide (environ $500 \mathrm{~ns}$ ) et non résolu dans le temps, est décrit par une interaction entre le récepteur et les micelles de CHAPS. L'ajout de holotransferrine (transferrine saturée en fer) à la solution fait apparaître un second mode de relaxation qui se manifeste par une augmentation exponentielle de l'émission de fluorescence en fonction du temps (figure 4A). Ce phénomène, qui se déroule en une cinquantaine de microsecondes, peut être décrit par les équations (1) à (6) de la relaxation chimique, où $A$ est l'holotransferrine, $B$ le récepteur et $C_{1}$ l'adduit. On vérifie bien que l'inverse du temps de relaxation mesuré dépend linéairement de la somme des concentrations en récepteur et en holotransferrine (figure 4B, équation 7).

$$
\begin{gathered}
\tau^{-1}=\mathrm{k}_{1}([\text { holotransferrine }]+[\text { récepteur }])+\mathrm{k}_{-1}= \\
\mathrm{k}_{1}([\mathrm{~A}]+[\mathrm{B}])+\mathrm{k}_{-1} \quad(7)
\end{gathered}
$$

Ce mode rapide est suivi par un troisième mode de relaxation très lent $(\sim 8000 \mathrm{~s})$, que nous ne montrerons pas ici. Ce processus cinétique est détecté par la variation de la différence d'absorption optique (absorption différentielle) entre un mélange de

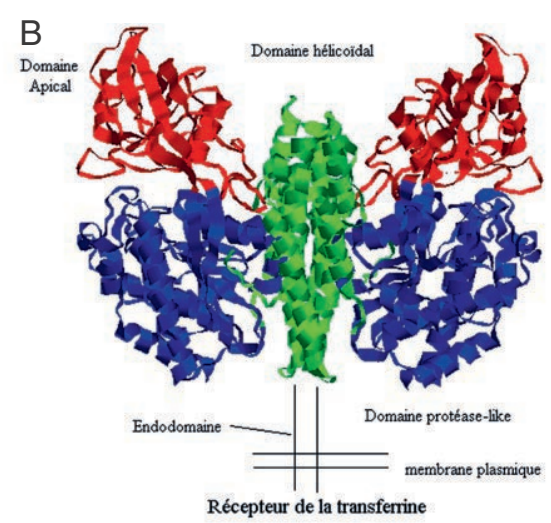



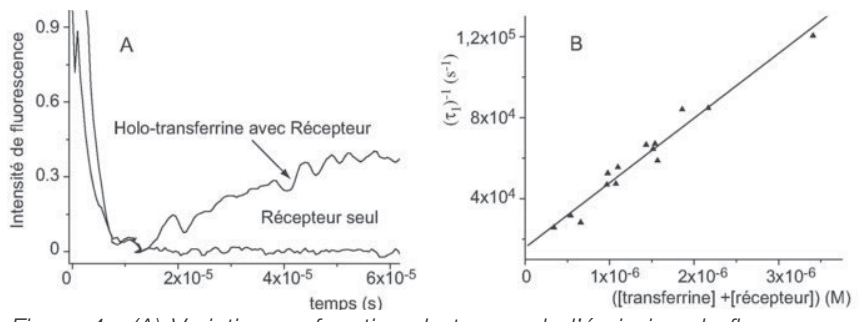

Figure 4: (A) Variation en fonction du temps de l'émission de fluorescence d'une solution de récepteur en présence de transferrine dans un milieu micellaire. La variation de l'intensité de fluorescence est proportionnelle à la variation des concentrations des espèces protéiques présentes dans le milieu réactionnel. (B) Variation des inverses des temps de relaxation associés au second phénomène rapide de la figure $4 \mathrm{~A}$ en fonction de la somme des concentrations en holotransferrine et en récepteur libre. La pente de la meilleure droite de régression est égale à $\mathrm{k}_{1}=3,2 \times 10^{10} \mathrm{M}^{-1} \mathrm{~S}^{-1}$ et l'ordonnée à l'origine est égale à $k_{-1}=1,6 \times 10^{4} \mathrm{~s}^{-1}$

récepteur et de holotransferrine avant et après réaction. Les inverses des temps de relaxation qui sont associés à ce phénomène lent sont indépendants de nos paramètres expérimentaux (équation 8)

$$
\tau_{2}^{-1}=k_{2}+k_{-2}
$$

L'analyse des trois modes de relaxation observés a permis de proposer un mécanisme pour l'interaction de la transferrine chargée en fer avec son récepteur, dans les milieux neutres en présence de micelles de CHAPS (réactions 9, 10 et 11, figure 5).

$\mathrm{B}+$ micelles $\rightleftharpoons$ adduit récepteur-micelles

(réaction ultra-rapide non résolue dans le temps)

$$
\begin{gathered}
\mathrm{A}+\mathrm{B} \underset{\mathrm{k}_{-1}}{\stackrel{\mathrm{k}_{1}}{\rightleftharpoons} \mathrm{C}_{1}} \\
\mathrm{C}_{1} \underset{\mathrm{k}_{-2}}{\stackrel{\mathrm{k}_{2}}{\rightleftharpoons} \mathrm{C}}
\end{gathered}
$$

Le récepteur $B$ interagit très rapidement avec la holotransferrine $A$ et donne un produit cinétique (complexe intermédiaire transferrine-récepteur) avec une constante de dissociation :

$$
\mathrm{K}_{1}=[\mathrm{A}][\mathrm{B}] /\left[\mathrm{C}_{1}\right]=\mathrm{k}_{-1} / \mathrm{k}_{1}=0,5 \mu \mathrm{M}
$$

Ce produit cinétique $\mathrm{C}_{1}$ subit alors un changement de conformation lent (8000 s) lui permettant d'atteindre son état thermodynamique $\mathrm{C}$, avec une constante d'équilibre :

$$
\mathrm{K}_{2}=[\mathrm{C}] /\left[\mathrm{C}_{1}\right]=\mathrm{k}_{2} / \mathrm{k}_{-2}=217
$$

Ce changement de conformation permet de mesurer une constante de dissociation globale pour l'adduit protéine-protéine :

$$
\mathrm{K}_{\text {globale }}=[\mathrm{A}][\mathrm{B}] /[\mathrm{C}]=\mathrm{K}_{1} / \mathrm{K}_{2}=2,3 \mathrm{nM} \text {. }
$$
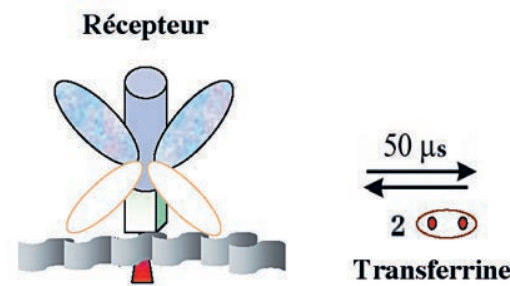

Intermédiaire cinétique
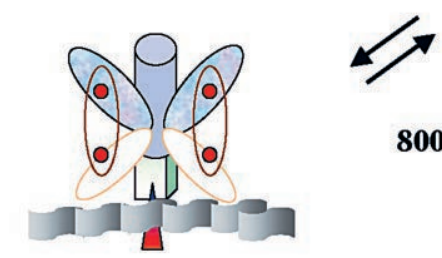

8000 s

Produit thermodynamique

Figure 5 : Mécanisme d'interaction entre la holotransferrine et le récepteur de la transferrine.

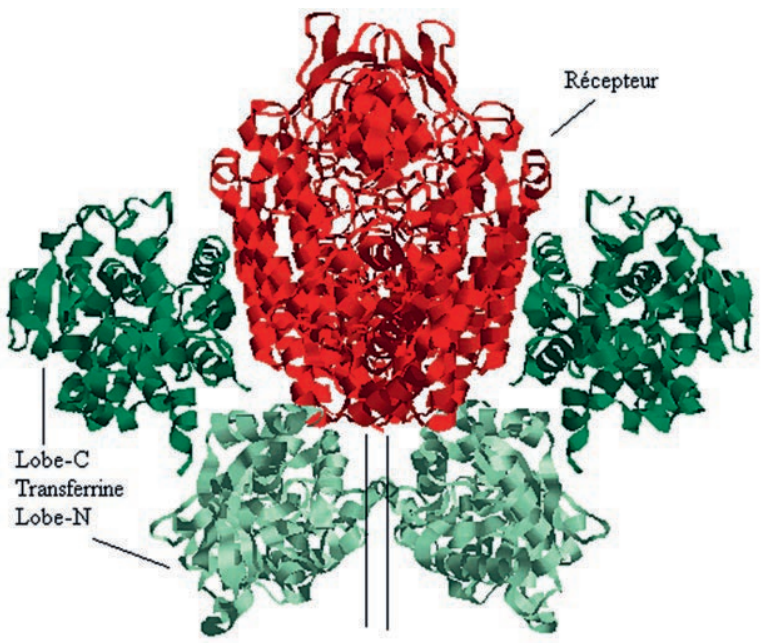

Adduit Holotransferrine-Récepteur

Figure 6 : Structure hypothétique de l'adduit dans son état thermodynamique $C$, formé par deux holotransferrines interagissant chacune avec une des deux sous-unités du récepteur de la transferrine.

Le changement de conformation du produit cinétique holotransferrine-récepteur va donc avoir pour effet de stabiliser l'adduit transferrine-récepteur d'un facteur de 217.

Ce mécanisme a été confirmé par des données obtenues par cryo-microscopie électronique, associée à la modélisation moléculaire, où il a été montré qu'il existe deux interactions différentes entre le récepteur et la holotransferrine. La première, forte, est celle du lobe $\mathrm{C}$ de la holotransferrine avec le domaine hélicoïdal du récepteur : la seconde, beaucoup plus faible, est celle où le lobe $\mathrm{N}$ vient s'insérer dans une gorge située entre la membrane et le récepteur (figure 6) [5]. Nous pouvons donc conclure que l'étape cinétique de 50 us (figure 4A) décrit l'interaction rapide du lobe $\mathrm{C}$ avec le domaine apical du récepteur, tandis que l'étape de $8000 \mathrm{~s}$ décrirait le changement de conformation de la transferrine, dont le lobe $C$ est en interaction avec le récepteur, pour permettre au lobe $\mathrm{N}$ de s'insérer entre le récepteur et la membrane plasmique (figure 6).

\section{Conclusion}

Cet exemple illustre une faible partie des nombreuses possibilités qu'offrent les méthodes et techniques de la relaxation chimique pour l'analyse de systèmes biologiques complexes multiréactionnels et réversibles. Pour que ces techniques soient applicables, deux conditions sont requises. (i) Le système doit être sensible au type de perturbation dont dispose l'expérimentateur (saut de température ou de concentration), et (ii) il faut également posséder un moyen de détection rapide permettant de suivre le réarrangement des concentrations des espèces présentes en fonction du temps (spectrophotométrie d'émission ou d'absorption). La littérature montre qu'il existe un très grand nombre de systèmes réactionnels de type " boîtes noires ", dont les mécanismes internes sont inconnus et peuvent être déterminés par les techniques et méthodes de la relaxation chimique.

\section{Références}

[1] Eigen, M. et DeMaeyer, L. (1963) «Relaxation methods ». Dans Techniques of Organic Chemistry - Investigation of Rates and Mechanism of Reactions, part II (Friess, S.L., Lewis, E.S. et Weissberger, A., eds), vol. 8, pp. 895-1029. Wiley Interscience, New-York.

[2] Williamson, J.C., Zewail, A.H. (1991). "Structural femtochemistry : experimental methodology", Proc. Natl. Acad. Sci. USA 88, 5021-5025.

[3] Hémadi M., Kahn, P.H., Miquel, G. et El Hage Chahine, J.M. (2004). "Transferrin's mechanism of interaction with receptor 1", Biochemistry 43, 1736-1745.

[4] Miquel, G., Nekaa, T., Kahn, P.H., Hémadi, M. et El Hage Chahine, J.M. (2004). "Mechanism of formation of the complex between transferrin and bismuth, and interaction with transferrin receptor 1", Biochemistry 43, 14722-14731.

[5] Cheng, Y., Zak, O., Alsen, P., Harrison, S.C., et Walz, T. (2004). "Structure of the human transferrin receptor-transferrin complex", Cell $116,565-576$ 